\title{
Données entomologiques préliminaires pour la mise en place d'une pulvérisation intra-domiciliaire à grande échelle dans la commune de Corpargo au Nord-Est du Bénin
}

\author{
Anges YADOULETON ${ }^{1,2^{*}}$, Rock AÏKPON ${ }^{1,2}$, Geraldo HOUNDETON ${ }^{2}$, \\ Sidick ABOUBACAR ${ }^{2}$, Fabrice URSINS ${ }^{2}$, Carine TCHIBçOZO $^{3}$, Achaz AGOLINOU ${ }^{3}$ \\ et Martin AKOGBETO ${ }^{2}$ \\ ${ }^{\text {I}}$ Ecole Normale Supérieure de Natitingou ; Université Nationale des Sciences, Technologies, Ingénierie et \\ Mathématiques, Bénin. \\ ${ }^{2}$ Centre de Recherche Entomologique de Cotonou, Bénin. \\ ${ }^{3}$ Laboratoire des Fièvres Hémorragiques, Bénin. \\ *Corresponding author, E-mail: anges33@yahoo.fr
}

\section{RESUME}

Au Bénin, la diversité bioclimatique du sud forestier au nord savanicole induit des faciès de transmission de paludisme très varié. Mais peu de travaux ont été réalisés sur la transmission du paludisme en zone de savane sèche au nord du pays. C'est dans ce cadre qu'une étude sur la variation saisonnière de la transmission du paludisme en prélude à la mise en place d'une Pulvérisation Intra Domiciliaire (PID) à grande échelle dans la commune de Corpargo au Nord-Est du Bénin a été initiée. Dans un premier temps, des captures de moustiques sur appât humain ont été effectuées dans deux villages à un rythme de 2 nuits consécutives par mois. Ces captures sur appât humain ont été complétées par des captures au pyrèthre effectuées à l'intérieur des maisons et ceci 2 fois par mois dans 10 habitations choisies également dans les mêmes zones de capture afin de connaître la diversité de la faune culicidienne. Dans un deuxième temps, le thorax des moustiques capturés sur appât humain a été utilisé pour rechercher la positivité en protéines Circum sporozoüte (CS) de Plasmodium falciparum par la méthode de titrage immunoenzymatique (ELISA-CSP). Cette étude a révélé la présence de plusieurs espèces de moustiques dont Anopheles gambiae s.s (30\% de la population collectée) avec une densité agressive moyenne mensuelle de 3,65 piqûres par homme et par nuit. Cette espèce de An. gambiae retrouvée est responsable de la transmission du paludisme tout au long de l'année. Cette étude montre une fois encore que An. gambiae s.s reste le principal vecteur de la transmission du paludisme avec de fortes densités agressives dans cette commune. Ces données de base seront d'une grande utilité dans la mise en place des stratégies de la PID, gage de la réduction du taux de mortalité dû au paludisme dans cette région.

(C) 2018 International Formulae Group. All rights reserved.

Mots clés: Anopheles gambiae, paludisme, transmission, Copargo, Benin. 


\title{
Preliminary entomological data for the implementation of insecticide residual spray in the district of Copargao, North-East of Benin
}

\begin{abstract}
In Benin, the bioclimatic diversity of the dry savannah induces different aspect of malaria transmission. But little has been known on malaria transmission in this ecosystem in Benin. Therefore, a cross sectional entomological study was carried out from January to December 2014 in the district of Corpargo in North-East of Benin in order to evaluate the level of malaria transmission base on entomological parameters. Heads and thoraces of Human Landing Catches (HLC) were performed monthly over two consecutive nights in 4 randomly selected compounds the Human Biting Rates (HBRs), complemented by Pyrethrum Spray Catches (PSC) in 4 other selected compounds. The same compounds and sampling methods were used consistently throughout the study. Mosquitoes from HLC catches were tested for the presence of Circum sporozoite Protein (CSP) of P. falciparum using an enzyme-linked immunosorbent assay (ELISA) to evaluate the sporozoite rate and Entomological Inoculation Rate (EIR). This study revealed several species of mosquitoes where Anopheles gambiae represented $30 \%$ of the mosquitoes caught. The HBRs of An. gambiae was 3.65 bites/person/night. This study showed also that malaria transmission is permanent through the year but intensive during the rainy season. These findings showed once again that the main malaria parasite Plasmodium falciparum was transmitted by Anopheles gambiae through the year. These results will be very important for the implementation of Insecticide Residual Spray strategies to reduce malaria intensity in this district.
\end{abstract}

(C) 2018 International Formulae Group. All rights reserved.

Keywords: Anopheles gambiae, malaria transmission, Copargo, Benin.

\section{INTRODUCTION}

Le paludisme reste l'une des premières causes de mortalité dans le monde et en Afrique subsaharienne (WHO, 2012). Parmi les maladies à transmission vectorielle, il constitue pour les hommes une menace constante de santé publique tant par la morbidité que par la mortalité qu'il entraîne (WHO, 2012). Cette maladie est due à la présence dans le sang d'hématozoaires du genre Plasmodium transmise par les piqûres infectantes de moustiques du genre Anopheles. Elle constitue un problème de santé publique pour plus de 3,5 milliards de personnes vivant dans 106 pays et territoires dans le monde (WHO, 2012). En 2014, environ 198 millions de cas et 584000 décès ont été enregistrés dans le monde dont plus de $80 \%$ surviennent en Afrique sub-saharienne (WHO, 2012).

Malgré les efforts nationaux et internationaux, la charge de la morbidité palustre reste élevée notamment dans les régions tropicales de l'Afrique. Au Bénin, le paludisme vient en tête des motifs de consultation et d'hospitalisation (Akogbéto et al., 2010). Son incidence dans le département de la Donga en 2013 était de 22,1\% dépassant largement la moyenne nationale qui est de 15,7\% (Aikpon et al., 2013). La transmission $\mathrm{du}$ paludisme en Afrique intertropicale est très hétérogène du fait des variations écoclimatiques (Tchuinkam et al., 2010). Elle est assurée par de nombreuses espèces anophéliennes de façon permanente, temporaire ou occasionnelle (Yadouleton et al., 2011). Ces anophèles sont souvent des groupes ou complexes d'espèces morphologiquement proches, colonisant des environnements différents et ayant des comportements trophiques et de repos très contrastés. La connaissance de la biologie des vecteurs de paludisme et de la détermination de leur rôle dans la transmission peuvent permettre l'amélioration 
des actions de lutte contre la maladie (Djenontin et al., 2010).

Au Bénin, la diversité bioclimatique du sud forestier au nord savanicole induit des faciès de transmission variés. Des travaux antérieurs dans le pays ont montré l'implication de Anopheles gambiae stricto sensu (Giles), de An. Arabiensis (Yadouleton et al., 2010) et de Anopheles funestus (Giles) (Djenontin et al., 2010) dans la transmission du paludisme. Mais peu de travaux ont été réalisés sur la transmission du paludisme en zone de savane sèche au nord du pays. Cette étude vise à répondre en partie à ce manque de données sur la transmission du paludisme dans cette zone au nord du Bénin afin de disposer des données entomologiques pour la mise en place à grande échelle de la PID dans la commune de Copargo.

\section{MATERIEL ET METHODE Zone d'étude}

L'étude a été menée au nord-ouest du Bénin dans le département de la Donga de janvier à novembre 2014. Ce département couvre une superficie de $11126 \mathrm{~km}^{2}$ et compte un total de 483085 habitants en 2012 (INSAE, 2012). Ce département est caractérisé par un climat tropical sec marqué par une saison sèche (décembre-mai) et une saison pluvieuse (juin-novembre). $\mathrm{La}$ végétation est faite de savane arborée, arbustive et herbacée souvent clairsemée sous l'influence de l'agriculture. La moyenne annuelle des précipitations est de $1300 \mathrm{~mm}$ et les températures moyennes mensuelles varient entre $23{ }^{\circ} \mathrm{C}$ et $32^{\circ} \mathrm{C}$. L'étude a été réalisée dans les villages de Kataban et de Kparakounan dans la commune de Copargo (Figure 1).

\section{Echantillonnage des populations de moustiques}

Le recueil des données a reposé sur un suivi entomologique longitudinal réalisé de janvier à novembre 2014 grâce à des captures de nuit sur sujets humains. Des captures mensuelles de moustiques agressifs ont été réalisées de $20 \mathrm{~h}$ à $6 \mathrm{~h}$ du matin, sur des sujets humains volontaires qui, au préalable, ont donné leur consentement favorable pour le déroulement de l'activité.

Dans la zone d'étude, les captureurs ont été placés à l'extérieur et à l'intérieur de 4 habitations par maison choisie comme points de captures, soit 16 captureurs par mois. La collecte s'est déroulée à un rythme de 2 nuits consécutives par mois. Ces lieux de capture n'ont pas été variés tout au long de l'étude. Les moustiques capturés ont été conservés individuellement dans des tubes à hémolyse bouchés avec du coton et gardés par tranche horaire et par point. Ces moustiques ont été identifiés le lendemain matin selon le genre et l'espèce à partir de la clé de Gillies et de Meillon (1968).

Tous ces moustiques ont été conservés sur du silicagel pour la recherche de l'infection par la méthode de titrage immunoenzymatique (Elisa CSP).

\section{Indices entomologiques de la transmission du paludisme}

En un lieu donné, plusieurs facteurs influencent la transmission du paludisme du moustique à l'homme. L'estimation quantitative de cette transmission se fait à travers un certain nombre d'indices mathématiques calculés à partir des données recueillies lors des enquêtes entomologiques.

\section{Densité agressive}

Encore appelée taux d'agressivité, elle est le produit de la densité anophélienne en relation avec l'Homme (m) et du taux d'anthropophilie (a). Elle s'exprime en nombre de piqûres d'anophèles par Homme par unité de temps. Elle s'obtient en divisant le nombre total d'anophèles capturés par le nombre de sujets utilisés, par unité de temps.

\section{Taux de parturité}

On obtient le taux de parturité d'une population anophélienne en calculant le 1995 
pourcentage de femelles pares (qui ont pondu au moins une fois) par rapport au nombre total des femelles examinées.

Une population anophélienne sera d'autant plus dangereuse épidémiologiquement que son taux de parturité sera élevé. En effet, seuls les anophèles pares sont susceptibles d'être infectantes.

$\mathrm{TP}=\mathrm{P} \times 100 /(\mathrm{P}+\mathrm{NP}) ; \mathrm{TP}=$ Taux de parturité ; $\mathrm{P}=$ nombre de femelles pares ; $\mathrm{NP}=$ nombre de femelles nullipares

\section{Taux d'infection des anophèles}

Le taux d'infection (S) est la proportion de moustiques infectés dans une population. Il s'exprime sous forme de pourcentage (nombre de moustiques infectés sur nombre de moustiques examinés fois cent).

\section{Taux d'inoculation entomologique (h)}

C'est le nombre de piqûres infectantes que reçoit un homme pendant une période donnée. C'est le produit du taux de piqûre par l'indice circumsporozoïtique (ou sporozoïtique).

$\mathbf{h}=\mathbf{m a} \times \mathbf{S} ; \mathbf{S}=$ indice sporozoïtique ; ma agressivité ;

\section{Traitement des moustiques sur le terrain}

Les moustiques ont été identifiés le lendemain matin suivant le genre et l'espèce à partir de la clé d'identification morphologique. Les Anophèles ont été dénombrés par lot horaire et par lieu de capture. Les ovaires des femelles de An. gambiae s.l ont été disséqués pour la détermination de la parturité suivant l'aspect des trachéoles ovariennes (Detinova et Gillies., 1964). Tous les anophèles vecteurs ont été conservés sur du silicagel dans des tubes eppendorfs à $-20{ }^{\circ} \mathrm{C}$ au laboratoire pour rechercher la positivité en protéine circum sporozoïte (C.S.) de P. falciparum.

\section{Traitement au laboratoire}

Les moustiques ont été découpés en 2 parties : tête-thorax et carcasse (abdomen, ailes, pattes). La partie tête-thorax a été analysée par ELISA (Enzym Linked Immunosorbent Assay) pour la recherche des antigènes circum Sporozoïtique de $P$. falciparum (Wirtz et al., 1987). Les carcasses des moustiques ont servi à la détermination des diverses espèces du complexe $A n$. gambiae à partir de la technique de PCR (Polymérase chain reaction) (Cohoue et al., 2004).

\section{Analyse des données}

La densité agressive (ma) a été exprimée en nombre de piqûres par homme par nuit. La densité agressive à l'intérieur et à l'extérieur, et le total (intérieur +extérieur) a été calculée. Les valeurs ont été moyennées pour calculer l'agressivité moyenne au cours de la saison sèche et de la saison pluvieuse. L'indice sporozoïtique (s) a été calculé comme la proportion de moustiques trouvés positifs à l'antigène CS sur le total testé. Le Taux d'Inoculation Entomologique (TIE) a été calculé selon la formule : TIE $=\mathrm{s} \times$ ma. Le TIE a été calculé de façon globale mais aussi en fonction des saisons (sèche et pluvieuse). Le taux de parturité a été exprimé comme le pourcentage des moustiques pares sur le total disséqué. Le taux de parturité et le TIE ont été comparés grâce à une régression binomiale négative. Le nombre de An. gambiae s.l. capturés à l'intérieur et à l'extérieur des habitations a permis de calculer le taux d'endophagie. Le test de $\mathrm{Chi}^{2}$ a permis de comparer l'agressivité anophélienne et le TIE suivant les saisons. 


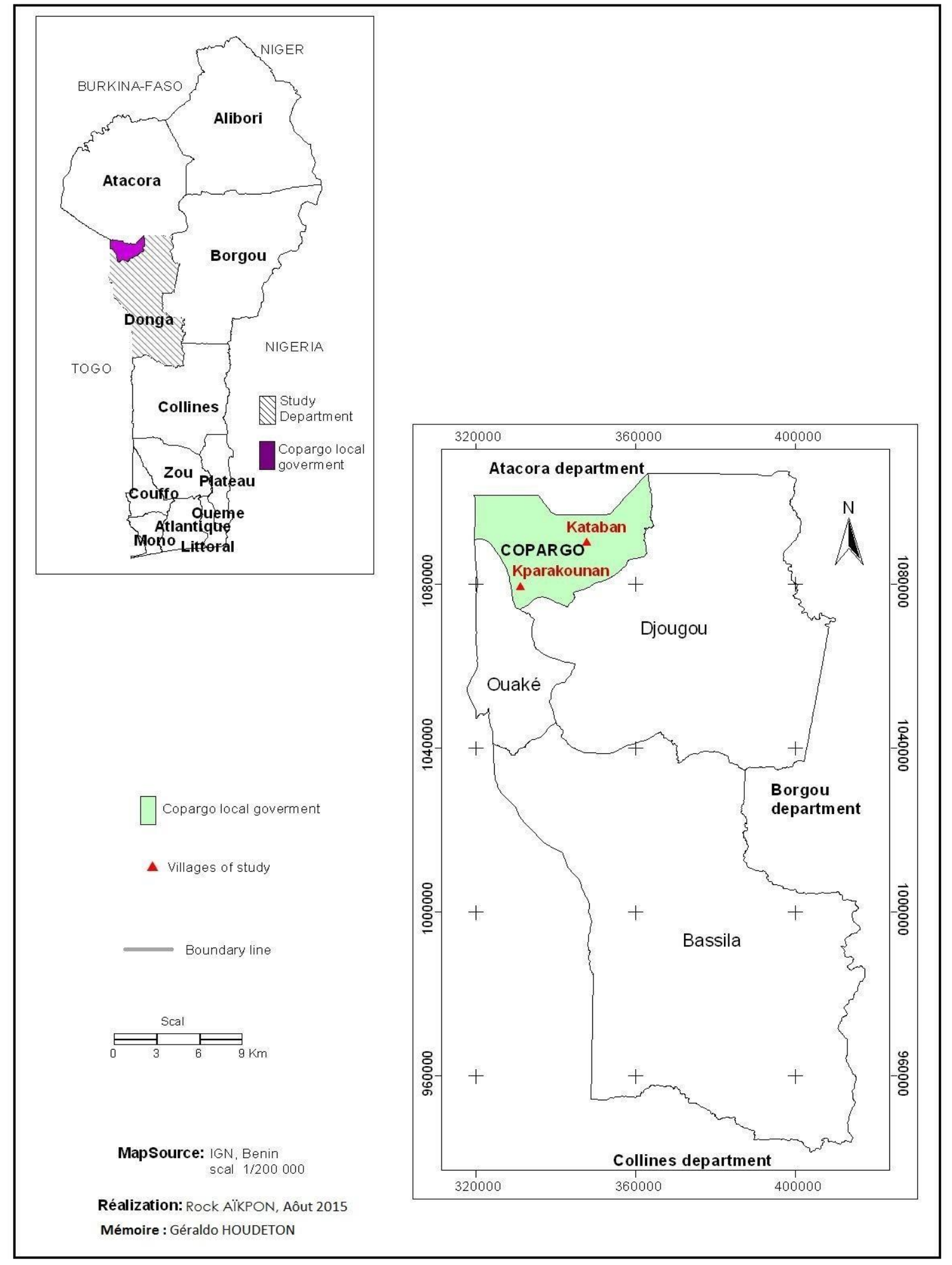

Figure 1 : Carte de la zone d'étude montrant les villages enquêtés. 


\section{RESULTATS}

\section{Faune anophélienne}

$\mathrm{Au}$ total, 853 moustiques ont été capturés durant 176 capture-nuits (88 capture-nuits à l'intérieur et à l'extérieur) entre janvier et novembre 2014. Ces moustiques sont constitués majoritairement d'espèces du genre Anopheles (81,48\%). An. gambiae s.l., Anopheles du groupe funestus, An. pharoensis, An. ziemanni, An. coustani ont été les principaux taxons rencontrés dans la localité. Parmi les anophèles, An. gambiae s.l est majoritaire soit $92.52 \%$ (643) (Tableau 1). De plus, des 643 An. gambiae collectés, $425(66,1 \%)$ sont capturés à l'intérieur des habitations, confirmant le caractère endophage de cette espèce.

\section{Taux agressivité de An. gambiae}

La Figure 2 présente la variation horaire de l'agressivité nocturne de $A n$. gambiae à l'intérieur et à l'extérieur des habitations. On note une agressivité plus élevée de An. gambiae entre $23 \mathrm{~h}$ et $05 \mathrm{~h}$ avec un pic aux environs de $01 \mathrm{~h}$ aussi bien à l'intérieur qu'à l'extérieur. En effet, $92,38 \%$ des piqûres surviennent après $23 \mathrm{~h}$. De janvier à novembre 2014, le taux moyen de piqûre par homme et par nuit est de 3,65 (4,83 à l'intérieur et 2,48 à l'extérieur). L'activité de piqûre de $A n$. gambiae à Copargo suivant la pluviométrie est présentée par la Figure 3 (A et B) avec un pic maximum de $8,13 \mathrm{p} / \mathrm{H}$ durant le mois de septembre.

La variation saisonnière montre que le taux moyen de piqûre est significativement plus important $(\mathrm{P}<0,05)$ en saison pluvieuse $(5,70 \mathrm{p} / \mathrm{h})$ qu'en saison sèche $(1 \mathrm{p} / \mathrm{h})$.

\section{Identification moléculaire}

Des 282 moustiques de An. gambiae échantillonnés de façon aléatoire du total collecté et analysé à la technique de la PCR, il révèle qu'ils étaient composés exclusivement de An. gambiae s.s.

\section{Taux d'inoculation entomologique et taux de parturité}

Tous les moustiques (An. gambiae et An. funestus) capturés ont été testés à l'ELISA CSP. Aucun positif n'a été trouvé au sein de la population de An. funestus. Chez An. gambiae, aucune différence significative de l'indice sporozoïtique n'a été observée entre la saison sèche et la saison pluvieuse. La Figure 4 montre l'évolution mensuelle du TIE de janvier à novembre 2014. On note un TIE annuel global de 182 piqures infectantes par personne par an (pi/h/an). Cependant, le TIE connaît une grande variation saisonnière. En effet, il est de 21 piqures infectantes par personne par mois (pi/h/mois) en saison pluvieuse contre 5 $\mathrm{pi} / \mathrm{h} /$ mois en saison sèche. La transmission du paludisme a été quasi permanente. La transmission maximale a été notée en juillet (1,31 $\mathrm{pi} / \mathrm{h} /$ nuit soit 39,3 $\mathrm{pi} / \mathrm{h} / \mathrm{mois})$.

Pour le taux de parturité, on note une grande variation suivant les saisons. Le taux de parturité en saison sèche est très élevé $(90,77 \%)$ contre $59,53 \%$ en saison des pluies. Aucune corrélation n'a été observée entre l'évolution du TIE et la longevité d'An. gambiae. 
Tableau 1: Diversité culicidienne des moustiques collectés à Copoargo par capture sur appât humain de janvier à novembre 2014 à l'intérieur et à l'extérieur des habitations.

\begin{tabular}{cccr}
\hline Espèces & Intérieur & Extérieur & Total \\
\hline An. Gambiae & 425 & 218 & 643 \\
An. Funestus & 3 & 1 & 15 \\
An. Pharoensis & 2 & 0 & 2 \\
An. Ziemanni & 5 & 5 & 10 \\
An. Coustani & 1 & 1 & 25 \\
Ae. Aegypti & 4 & 6 & 10 \\
Ae. Vittatus & 1 & 1 & 2 \\
Cx. Quinquefasciatus & 5 & 6 & 118 \\
Mansonia africana & 4 & 1 & 21 \\
Mansonia uniformis & 1 & 6 & 7 \\
\hline Total & $\mathbf{5 0 7}$ & $\mathbf{3 4 6}$ & $\mathbf{8 5 3}$ \\
\hline
\end{tabular}

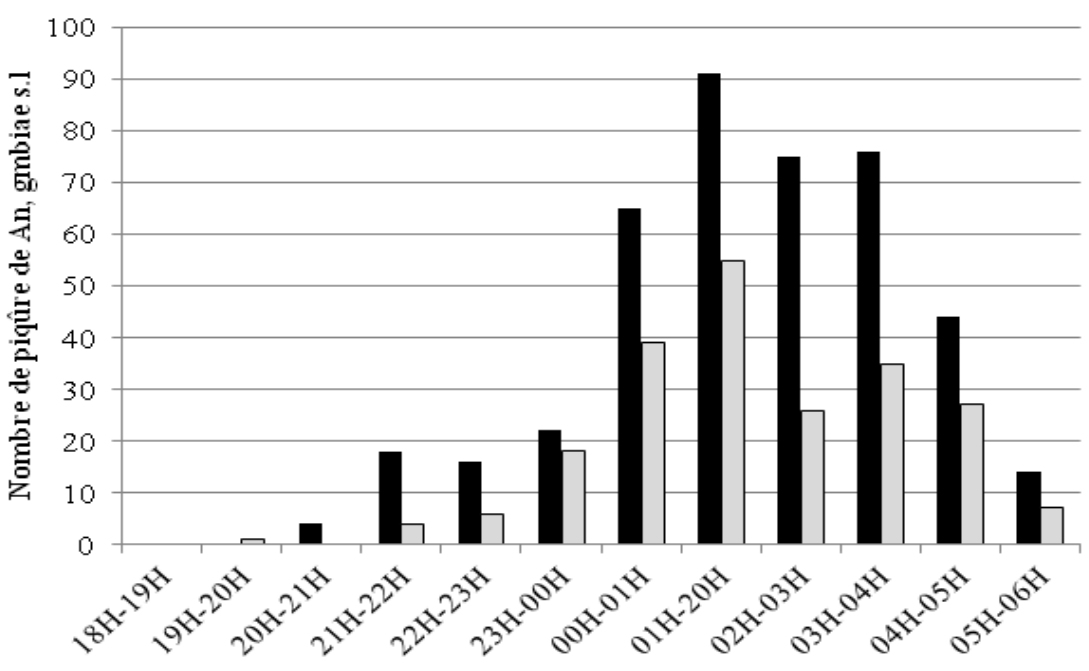

Figure 2 : Distribution horaire du nombre de piqure An.gambiae s.l à l'intérieur et l'extérieur des habitations à Copargo de janvier à novembre 2014. 


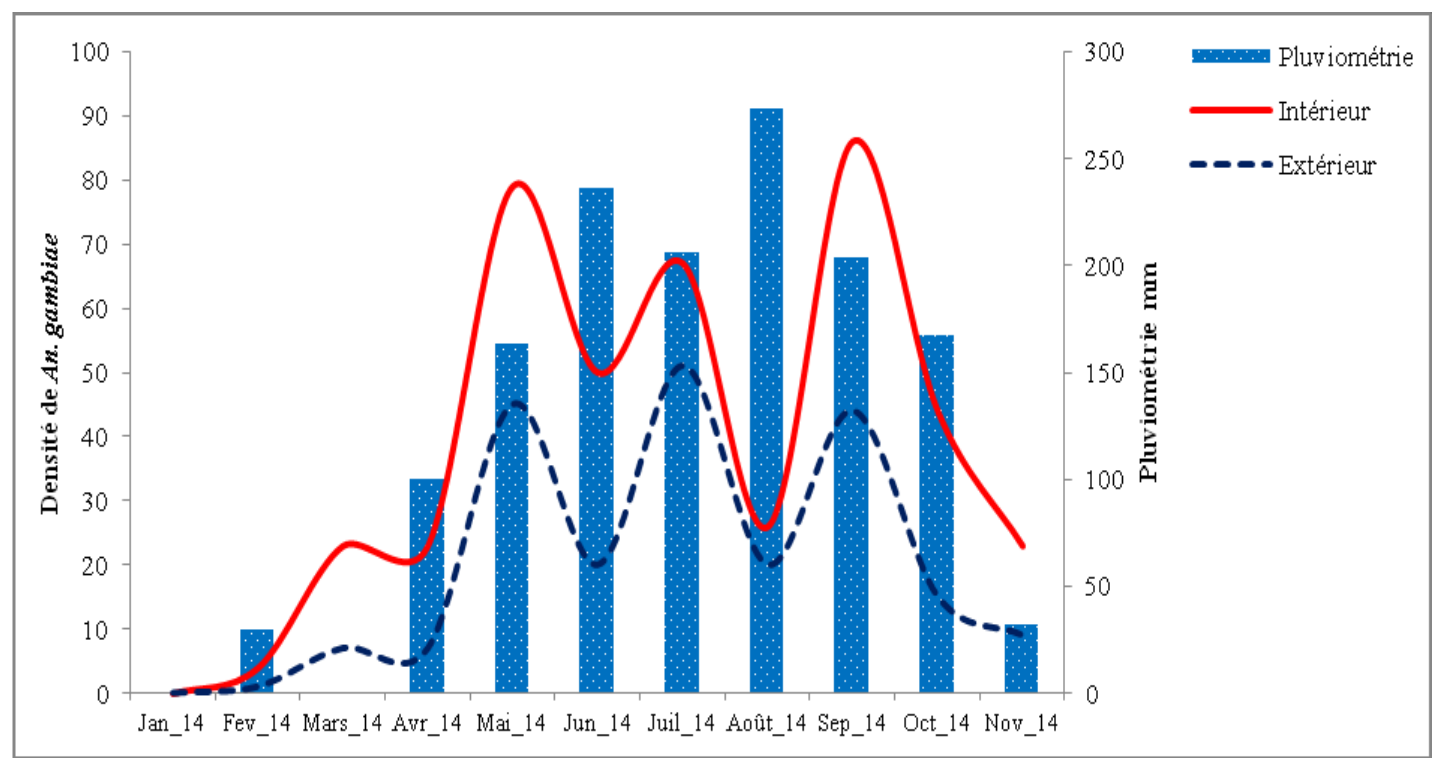

Figure $3 \mathrm{~A}$ : Densité de An. gambiae à l'intérieur et l'extérieur des habitations en fonction de la pluviométrie de janvier à novembre 2014 à Copargo.

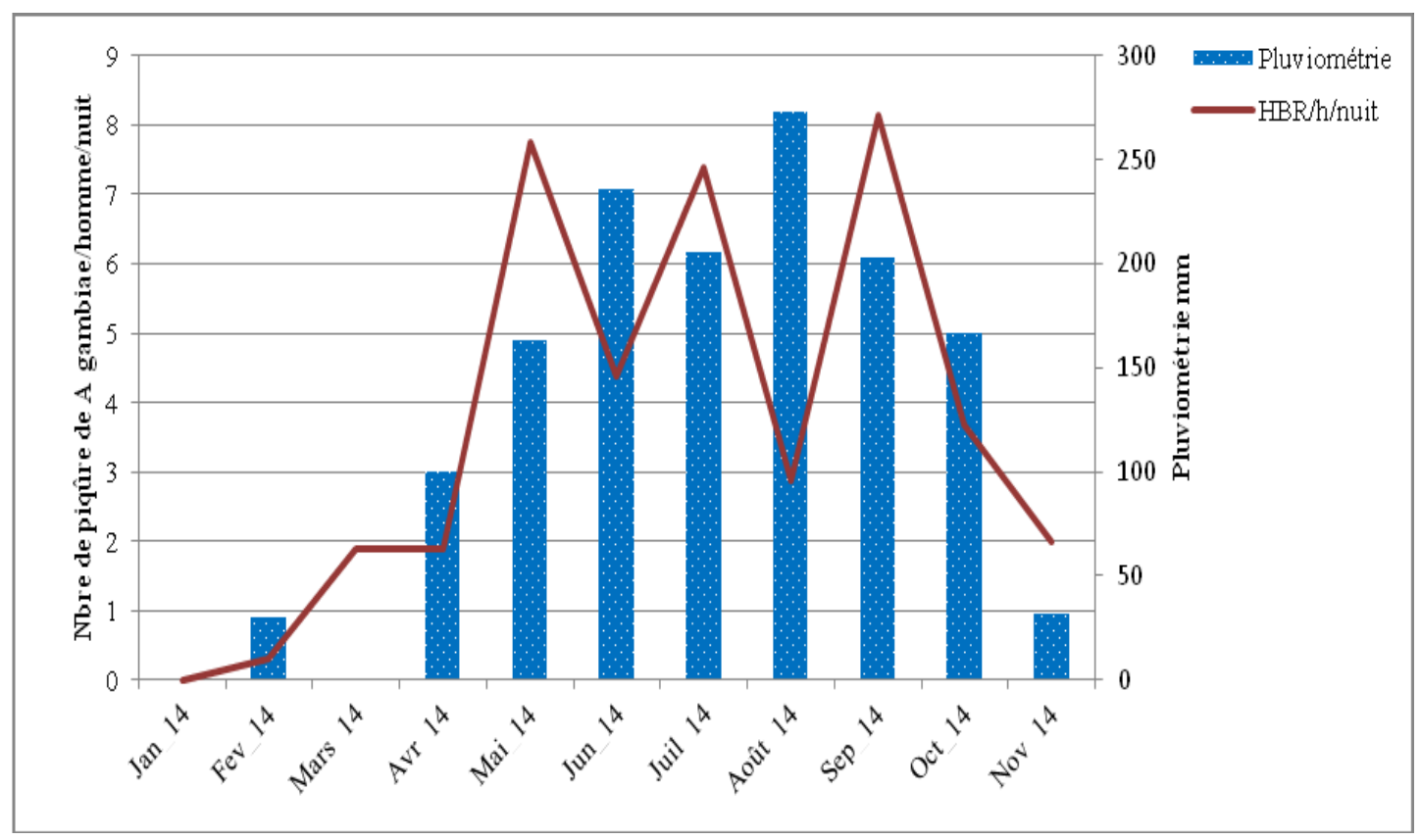

Figure 3 B: Nombre de piqûre de An. gambiae/homme /nuit en fonction de la pluviométrie. 


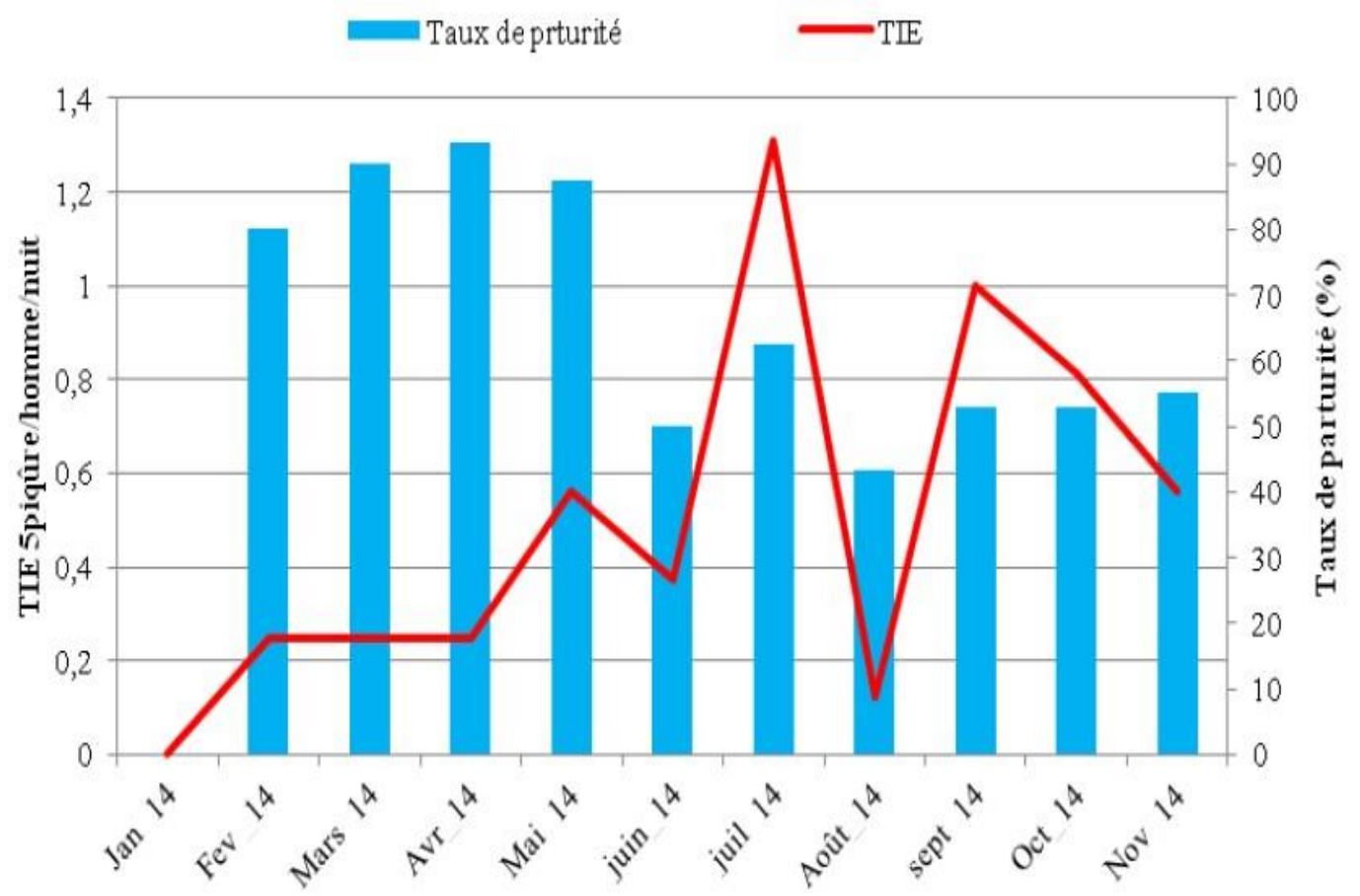

Figure 4 : Evolution mensuelle du taux d'inoculation entomologique et de la longévité de janvier à novembre 2014.

\section{DISCUSSION}

L'étude des moustiques vecteurs et de la dynamique de la transmission du paludisme est un préalable indispensable non seulement pour comprendre l'épidémiologie de la maladie mais aussi pour mettre en place un contrôle efficace et ciblé de ces vecteurs (Fontenille et al., 2004). L'étude qui à été réalisée dans deux villages de la commune de Copargo dans le département de la Donga au nord-ouest du Bénin avait pour but d'identifier les vecteurs présents et d'estimer le niveau de transmission du paludisme. Ces données obtenues permettront de mettre en place des stratégies pour les activités d'aspersion intra-domiciliaire à grande échelle ceci, pour la baisse de la transmission du paludisme dans cette localité.

Le suivi entomologique longitudinal, réalisé pendant onze mois dans ces villages nous a permis d'identifier cinq espèces anophéliennes dont deux déjà incriminées dans la transmission du paludisme au Bénin (Aïkpon et al., 2013). Cette diversité d'espèce peut s'expliquer par la combinaison des différents facteurs écologiques et climatiques qui ont façonné des biotopes offrant des gîtes spécifiques au développement des larves de chaque espèce: cours d'eau permanent, flaques d'eau, petits barrages.

Un seul membre du complexe $A n$. gambiae a été retrouvé dans la zone d'étude. Il s'agit de An. gambiae s.s. Par ailleurs, la densité anophélienne augmente considérablement pendant la saison pluvieuse et cette augmentation est en relation avec la pluviométrie. Ce résultat confirme les travaux de Klinkenberg et al. (2008) au Ghana et de Okono et al. (2015) au Cameroon où ces auteurs ont montré que la densité anophélienne est fonction de la saison. Chaque pic ou une diminution de la pluviométrie a été responsable d'une augmentation ou une diminution de la 
population de An. gambiae s.l.. Les mois de mai, juillet et septembre sont les mois où les populations reçoivent plus de piqûres. L'agressivité à l'intérieur des habitations est plus élevée qu'à l'extérieur, ce qui pourrait s'expliquer par le comportement endophage de An. gambiae.

L'étude de l'infectivité au $P$. falciparum montre qu'aucun individu infecté n'a été retrouvé au sein de la population de An. funestus. Ceci serait probablement dû au faible effectif testé. La transmission du paludisme est assurée principalement par An. gambiae s.s., et ceci pendant toute l'année avec un TIE global moyen de $1 \mathrm{pi} / \mathrm{h}$ tous les deux jours. Ce taux est supérieur à ceux obtenus au sud du Bénin (Djenontin et al., 2010) et au nord-est du Bénin (Yadouléton et al., 2010). Toutefois, cette transmission connaît une variation saisonnière avec un niveau de transmission quatre fois plus élevé en saison pluvieuse qu'en saison sèche $(21 \mathrm{pi} / \mathrm{h} / \mathrm{mois}$ en saison pluvieuse contre $5 \mathrm{pi} / \mathrm{h} / \mathrm{mois}$ en saison sèche).

Le cycle d'agressivité des anophèles montre que $92,38 \%$ des piqûres surviennent après $23 \mathrm{~h}$ avec un pic aux environs de 01h. Ceci interpelle le Programme National de Lutte contre le Paludisme (PNLP) au renforcement des mesures de protection contre le paludisme telles que les moustiquaires imprégnées et la pulvérisation intra domiciliaire d'insecticide.

Le taux de parturité global moyen est de $65,85 \%$. Ce taux est plus élevé en saison sèche qu'en saison pluvieuse. En effet, lorsque la densité anophélienne est élevée, le taux de parturité est faible. Le fort taux de parturité observé pendant la saison sèche indique une longévité d'An. gambiae, ce qui augmenterait sa capacité vectorielle lui permettant de transmettre le paludisme pendant une période relativement longue. Ceci expliquerait le maintien de la transmission pendant la saison sèche. Cependant, aucune relation n'a été établie entre le taux de parturité et la longévité des vecteurs $(P=0,9)$.

\section{Conclusion}

L'objectif de cette étude est de recueillir des données entomologiques préliminaires en prélude à la mise en place de la stratégie de la pulvérisation intradomiciliaire à grande échelle pour lutter contre le paludisme dans la commune de Copargo au Nord-Est du Benin. Cette étude a montré que la transmission du paludisme a été intense et quasi permanente dans la zone d'étude. An. gambiae s.s. et An. funestus sont les deux vecteurs majeurs qui s'y retrouvent avec une prédominance de An. gambiae s.s. Les présents résultats informent sur la dynamique saisonnière de la transmission du paludisme et seront d'une grande utilité pour la prise de décision en matière du choix de lutte anti-vectorielle contre le paludisme dans la commune de la Copargo.

\section{CONFLIT D'INTERETS}

Les auteurs déclarent qu'ils n'ont pas de conflit d'intérêts.

\section{CONTRIBUTIONS DES AUTEURS}

$\mathrm{AY}, \mathrm{RA}$ et $\mathrm{GH}$ ont participé à l'élaboration du protocole, supervisé sa mise application et la rédaction de l'article. SA, FU, CT, AA ont participé à la collecte des données. MA a supervisé tout le travail et la rédaction du document final.

\section{REMERCIEMENTS}

Les auteurs du présent manuscrit remercient le Directeur de l'Ecole Normale Supérieure de Natitingou pour avoir financé ce travail de recherche.

\section{REFERENCES}

Aïkpon R, Agossa F, Ossè R, Oussou O, Aïzoun N, Oké-Agbo F, Akogbéto M. 2013. Bendiocarb resistance in Anopheles gambiae s.l. populations from Atacora department in Benin, West Africa: a threat for malaria vector control. Parasites \& Vectors, 6: 192. DOI: doi.org/10.1186/1756-3305-6-192

Akogbéto M, Padonou GG, Gbénou D, Irish S, Yadouleton A. 2010. Bendiocarb: a 
potential alternative against pyrethroid resistant Anopheles gambiae in Benin, West Africa. Malaria Journal, 9: 204210. DOI: doi.org/10.1186/1475-2875-9204

Akono Ntonga P, Tonga C, Mbida J, Ngo Hondt O, Awono-Ambene $\mathrm{P}$, Ndo C, et al. 2015. Anopheles gambiae, vecteur majeur du paludisme à Logbessou, zone péri-urbaine de Douala (Cameroun). Bull Soc Pathologie Exotique, 108: 360-368. DOI: $10.1007 / \mathrm{s} 13149-015-0452-3$.

Cohuet A, Simard F, Wondji C, AntonioNkondjio C, Awono-Ambene P, Fontenille D. 2004. High malaria transmission intensity due to Anopheles funestus (Diptera: Culicidae) in a village of savannah-forest transition area in Cameroon. J Med Entomol., 41: 901905. DOI: 10.1603/0022-2585-41.5.901

Detinova TS, Gillies MT. 1964. Observations on the Determination of the Age Composition and Epidemiological Importance of Populations of Anopheles gambiae Giles and Anopheles funestus Giles in Tanganyika. Bull World Health Organ. 30:23-28.

Djènontin A, Sahabi B, Moiroux N, Henry MC, Bousari O, Chabi J, Ossè R, Koudénoukpo S, Corbel V, Akogbéto M, Chandre F. 2010. Culicidae diversity, malaria transmission and insecticide resistance alleles in malaria vectors in Ouidah Kpomasse-Tori district from Benin (West Africa): A pre-intervention study. Parasites \& Vectors, 3: 83. DOI: 10.1186/1756-3305-3-83

Fontenille D, Simard F. 2004. Unravelling complexities in human malaria transmission dynamics in Africa through a comprehensive knowledge of vector populations. Comp Immunol Microbiol Infect Disease, 27: 357-375. DOI: 10.1016/j.cimid.2004.03.005

Gillies M, De Meillon B. 1968. The Anophelinae of Africa South of the Sahara (Ethiopian Zoogeographical Region). Publication of the South Afr Inst Med Res., 54: 1-343.

INSAE. 2012. Projection démographique révisée. Donga; 29.

Klinkenberg E, McCall PJ, Michael DW, Amerasinghe FP, Donnelly MJ. 2008. Impact of urban agriculture on malaria vectors in Accra, Ghana. Malaria Journal, 7: 151. DOI: 10.1186/14752875-7-151

Tchuinkam T, Simard F, Lélé-Defo E, TénéFossog B, Tateng-Ngouateu A, AntonioNkondjio C, et al. 2010. Bionomics of anopheline species and malaria transmission dynamics along an altitudinal transect in western Cameroon. BMC Infect Disease, 10:119. DOI: 10.1186/1471-2334-10-119

WHO. 2012. World Malaria Report. World Health Organization, Geneva.

Wirtz RA, Zavala F, Charoenvit Y, Campbell GH, Burkot TR, Schneider I, Esser KM, Beaudoin RL, Andre RG. 1987. Comparative testing of monoclonal antibodies against Plasmodium falciparum sporozoites for ELISA development. Bull World Health Organ., 65: 39-45.

Yadouléton AW, N'Guessan R, Allagbé H, Asidi A, Boko M, Osse R, G. Padonou, Gazard K, Akogbéto M. 2010. The impact of the expansion of urban vegetable farming on malaria transmission in major cities of Benin. Parasites \& Vectors, 3: 118. DOI: 10.1186/1756-3305-3-118. 